\title{
Real Time Simulation of Distribution System with Distributed Energy Resources
}

\author{
Tran Thai Trung, Seon-Ju Ahn, and Joon-Ho Choi
}

\begin{abstract}
This paper presents a real - time simulation of distribution system with distributed generation - DGs, Step voltage regulation (SVR) and battery energy storage systems (BESSs) using Real Time Digital Simulation (RTDS). The simulation models of a DG and a Nickel - Metal Hydride battery are developed as RSCAD user defined model. The control schemes of DGs and BESSs to show the impacts of DGs to local voltages and BESS to accommodate the variation of DG power output are proposed and implemented as a function block in the RSCAD platform. Difficulties of the system implementation in RTDS are also discussed and solutions are provided. Finally, the simulation results are given out to verify the correctness of the control schemes.
\end{abstract}

Index Terms-RTDS, distributed energy resource, battery energy resource, voltage control, user defined model.

\section{INTRODUCTION}

The penetration of the distributed energy resources in the transmission and distribution grids has been increasing, because of the limited supply of natural resources such as petroleum. The presence of DGs opens opportunities and poses new challenges. DGs can effectively reduce power losses and on-peak operation costs, improve voltage profile, defer system upgrades, and improve system integrity, reliability and efficiency with sophisticated control scheme [1]. However, the addition of DG may complicate protection coordination and voltage control. If not properly handled, lower reliability and even a reduction in power quality can result [2].

Generally, the voltage at a substation in distribution systems can be regulated within a predetermined range by an under-load tap changer (ULTC) [3]. In [4], a system to control the ULTC transformer voltages to ensure multiple feeder voltages remain within limits is proposed. However, if the DGs are installed in one of the multiple feeders, the voltage control might be failed with the ULTC control only.

Since DGs has stochastic nature, the energy storage systems (ESS) have been introduced to distribution systems to accommodate the variation of such power penetrations. The impact of energy storage costs on economic performance of a distribution substation was studied in [5], where the benefit and cost of installing an ESS were evaluated. A battery energy storage system (BESS) is the most commonly used ESS. It

Manuscript received November 25, 2013; revised January 16, 2014. This work was supported by Korea Institute of Energy Technology Evaluation \& Planning (KETEP) grant funded by Korea government Ministry of Trade, Industry \& Energy (No. 20123010020080) and the BK 21 PLUS project.

The authors are with Dept. of Electrical Engineering, Chonnam National University, Korea (e-mail: trantrung1811@gmail.com, sjahn@jnu.ac.kr, joono@jnu.ac.kr). requires a power conditioning system (PCS) which permits both active and reactive power to be generated. A PCS has high installation costs, and therefore its capacity should be fully utilized.

In order to study the effect of DGs and BESSs on the distribution system and to develop a novel control scheme of each voltage control devices, a detailed simulation model of each device must be performed. Since developing the multiple PV systems and modeling the distribution networks in the lab is relatively costly and time-consuming. The real time simulation therefore, will be the best solution for this problem. Real Time Digital Simulation (RTDS), which is widely used for real time simulations, provide a good tool to study the multiple DG systems.

This paper focuses on the process to develop the user defined model of the voltage control devices, DG and BESS to show their impacts on voltage control in distribution systems. It is organized as follows. Section II emphasizes on the distribution system modeling with RTDS, with the configuration process of each component in detail. In Section III, some control schemes of individual DG and the cooperation of DG and BESS in distribution system are presented. The conclusion is presented in Section IV.

\section{DISTRIBUTION SYSTEM MODEL}

\section{A. Distribution System Description}

The model with single line diagram as shown in Fig. 1 has been used for the simulation in this work. The model consists of a 4-MVA load located on the left side of an SVR, and 8 distributed 0.5-MVA loads on the right side. The SVR was assumed to be installed at the middle point of $18 \mathrm{~km}$ distribution feeder. All loads are worked at 0.9 power factor (p.f).

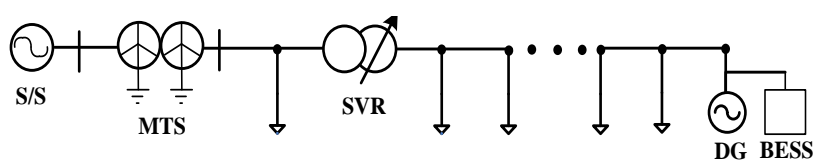

Fig. 1. Single line diagram of power system including DG.

With an objective of demonstrating the effect of the DG to voltage control and solving a real time industrial problem, a DG is being connected at the end of the feeder. A BESS is also connected near DG to deal with power curtailment. The simulation model of DG and BESS are described on the next section.

\section{B. Distribution System Description}

In order to test the effect of DGs to system voltage, this paper represents a simplified user defined model (UDM) for 
DGs by using current source and mathematics control model [6] as shown in Fig. 2.

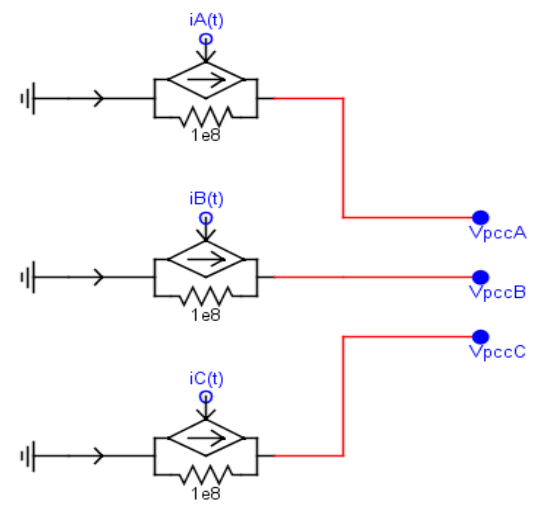

Fig. 2. DG model with controller.

Model is considered by equation (1) [7], as follows:

$$
S_{D G}=P_{D G} \pm j Q_{D G}=V_{P C C} \cdot I_{D G}^{*}
$$

where:

$P_{D G} \pm j Q_{D G}$ is the active and reactive power of DGs. ("+" sign mean DG compensates reactive power to grid, "_" sign mean DG absorbs reactive power from grid).

$V_{P C C}$ is the voltage at the point of common coupling (PCC).

From equation (1), we have:

$$
\begin{aligned}
S_{D G} & =P_{D G} \pm j Q_{D G}=\left|V_{P C C}\right| \angle \theta_{v} \cdot\left|I_{D G}\right| \angle-\theta_{i} \\
& \rightarrow\left|I_{D G}\right| \angle-\theta_{i}=\frac{P_{D G} \pm j Q_{D G}}{\left|V_{P C C}\right| \angle \theta_{V}}
\end{aligned}
$$

The current representing power output of DG can be calculated from referenced power values and measured voltage at the PCC. Thus, DG can be modeled as the current source with the reference signals calculated from equation (2).

Reference current signals of current source $i_{A}(t), i_{B}(t)$ and $i_{C}(t)$ are calculated individually for each phase. Each time active and reactive power of the DG changes, the controller calculates and adjusts the new reference value for current sources, to keep the output of DG is constant.

Fig. 3 shows the change of actual power of DG due to the change of the reference power.
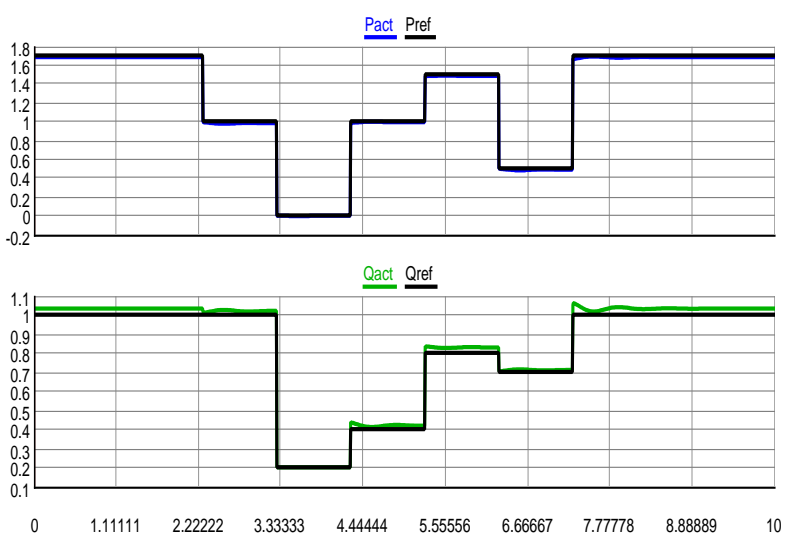

Fig. 3. Experiment comparison between reference and actual power of DG

From the Fig. 3, each time the reference values of active and reactive power change, the actual values at the output of DG also change with a very small error. This error appears because of the approximation in mathematics model of DG.

The control model of the DG used in this work is shown in Fig. 4.

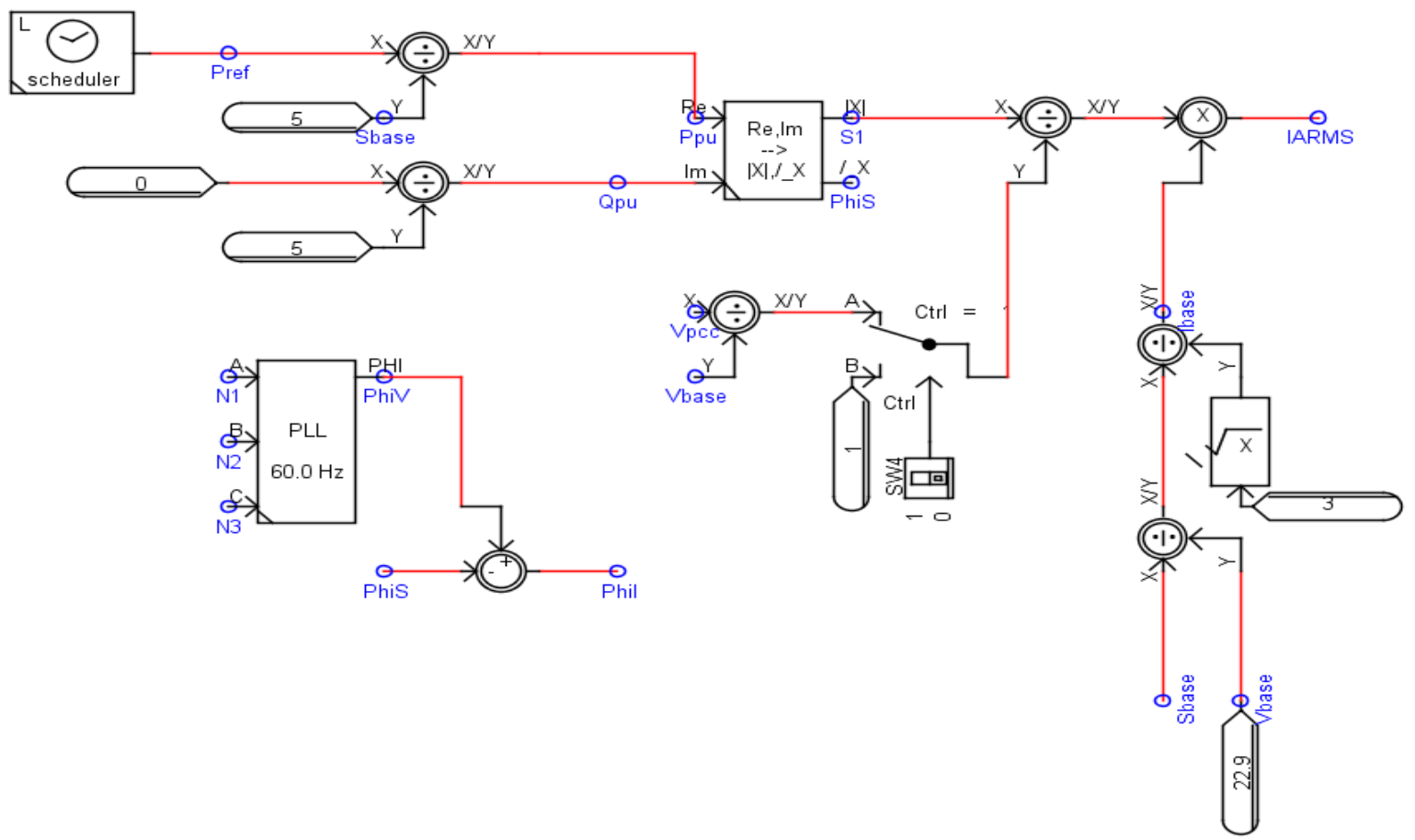

Fig. 4. DG control model.

\section{BESS Model}

In this paper, a Shepherd based model of Ni-MH battery is made by using Cbuilder software module in RSCAD environment. This model includes an equation to describe the electrochemical behavior of a battery directly in terms of terminal voltage, open circuit voltage, internal resistance, discharge current and state-of-charge [8]-[10]. The charge and discharge equations are summarized as follows: 
- Discharge:

$$
V_{\text {batt }}=E_{0}-R \cdot i-K \frac{Q}{Q-i t} \cdot\left(i t+i^{*}\right)+\operatorname{Exp}(t)
$$

- Charge:

$$
V_{\text {batt }}=E_{0}-R . i-K \frac{Q}{|i t|-0.1 . Q} \cdot i^{*}-K \frac{Q}{Q-i t} . i t+\operatorname{Exp}(t)
$$

where:

$V_{\text {batt: }}$ : battery voltage (V)

$E_{0}$ : battery constant voltage $(\mathrm{V})$

$K$ : polarization constant (V/Ah) or polarization resistance $(\Omega)$

$Q: \quad$ battery capacity (Ah)

it: $\quad$ actual battery charge (Ah)

A: $\quad$ exponential zone amplitude (V)

$B: \quad$ exponential zone time constant inverse $(\mathrm{Ah})^{-1}$

$R: \quad$ internal resistance $(\Omega)$

$i$ : $\quad$ battery current (A)

$i^{*}: \quad$ filtered current (A)

The user can use this model to visualize the charge and discharge curve obtained with the parameters and compare with that of the manufacturer.

Fig. 5 shows the test circuit for battery model. The block "Ni-MH battery stacks" represents not only one individual battery, but also the numerous batteries connected in parallel or series.

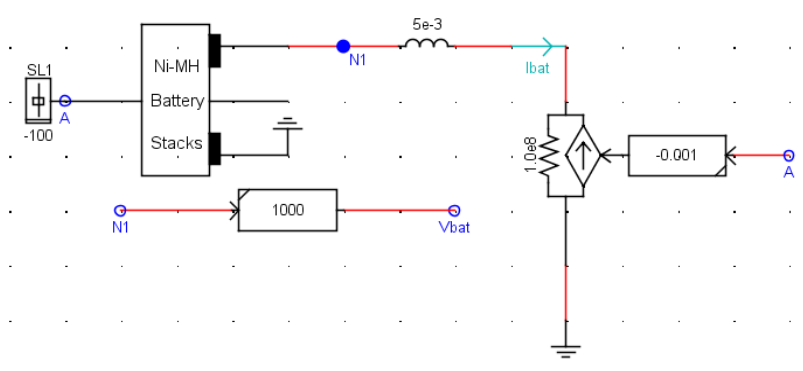

Fig. 5. Scheduler configurations.

Fig. 6 represents the result for the battery discharge simulation at $1 \mathrm{C}$. The result shows that this model can simulate actual Ni-MH battery correctly.

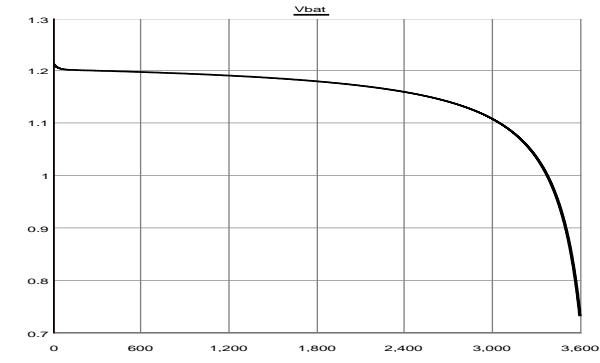

Fig. 6. Discharge curve (1C).

The main configuration of Ni-MH battery is presented in Fig. 7. This configuration interface can be modified by Cbuilder module to adapt with different kinds of battery or mathematic model used in simulation.

Because in RTDS, the main network is solved with a normal time - step size of about $50 \mu \mathrm{sec}$, whereas the voltage source converter (VSC) circuit (such as buck-boost converter) is solved with a time-step of 1.4 to $2.5 \mu \mathrm{sec}$, we must have an interface to connect a DC output of Ni-MH battery to main
AC grid.

In order to build a small time step simulation case for BESS, the first thing that must be done is to add a special hierarchy box into the RTDS simulation environment [11]. This special hierarchy box is known as a VSC Bridge box. Inside this box, the VSC circuit including firing pulse block to convert DC output of BESS and three-phase VSC interface transformer to connect small time step part with normal time step part should be added. The detail configuration of BESS system will be

\begin{tabular}{|c|c|c|c|c|c|}
\hline \multirow{2}{*}{\multicolumn{2}{|c|}{\begin{tabular}{|l|} 
CONFIGURATION \\
\end{tabular}}} & \multicolumn{4}{|c|}{ Ni-MH.def } \\
\hline & & Battery parameters & \multicolumn{2}{|c|}{ Battery array } & \\
\hline Name & \multicolumn{3}{|c|}{ Description } & Value & Unit \\
\hline E0 & \multicolumn{3}{|c|}{ Battery Constant voltage } & 1.26 & Jv \\
\hline K & \multicolumn{3}{|c|}{ Polarazation Constant } & 0.0001204 & $\mathrm{v}$ \\
\hline Q & \multicolumn{3}{|c|}{ Battery capacity } & 105.285 & Ah \\
\hline A & \multicolumn{3}{|c|}{ Exponential Zone Amplitude } & 0.1154 & \\
\hline$B$ & \multicolumn{3}{|c|}{ Exponential Zone Time Constant Inverse } & 0.1108 & Ah-1 \\
\hline $\mathrm{R}$ & \multicolumn{3}{|c|}{ Internal Resistance } & 0.0004576 & Ohm \\
\hline iVbat & \multicolumn{3}{|c|}{ initial battery voltage } & 1.39 & $\mathrm{~V}$ \\
\hline
\end{tabular}
discussed in the next section.

Fig. 7. Battery parameter configuration.

\section{BESS Model}

There is a two rack system based RTDS in our RTDS research lab, which has one WIF (Work Station Interface) card, one IRC (Inter Rack Communication) card, one GPC card with a GTDI (Giga Transceiver Digital Input) in each rack. In addition, one 3PC card with DDAC (Digital Analogue Converter) card is on the rack 2.

In order to test the real time performance of the DG controller, the control system is simulated in large time step in GPC card. The objectives of this controller are to provide voltage regulation and power factor correction. The simulation model for the controller has been built in RSCAD using its library components. The model of BESS is made by using Cbuilder program in RTDS, and connected to main grid through VSC interface. Fig. 8 illustrates the power system network with DG model in RSCAD environment.

\section{THE CONTROL SCHEMES}

\section{A. DG Voltage Control}

The voltage variation at the PCC due to the DG's active and reactive power output changes can be written as (5), where $R$ and $\mathrm{X}$ are the line resistance and reactance.

$$
\Delta \mathrm{V}_{\mathrm{PCC}} \approx \mathrm{P}_{\mathrm{DG}} \mathrm{R}+\mathrm{Q}_{\mathrm{DG}} \mathrm{X}
$$

In distribution system, the ratio of the line resistance to the reactance is not negligible. Therefore, the active power output of a DG raises the voltage at the PCC significantly. In this case, the function of reactive power output is to compensate voltage rise due to the effect of active power output. The above mentioned voltage control scheme is summarized in two simulations as follows:

Case 1: $Q_{D G}=0$.

In this case, the amount of voltage rise due to the active power output of a DG is:

$$
\Delta \mathrm{V}_{\mathrm{PCC}} \approx \mathrm{P}_{\mathrm{DG}} \mathrm{R}
$$

The simulation result is presented in Fig. 9. 


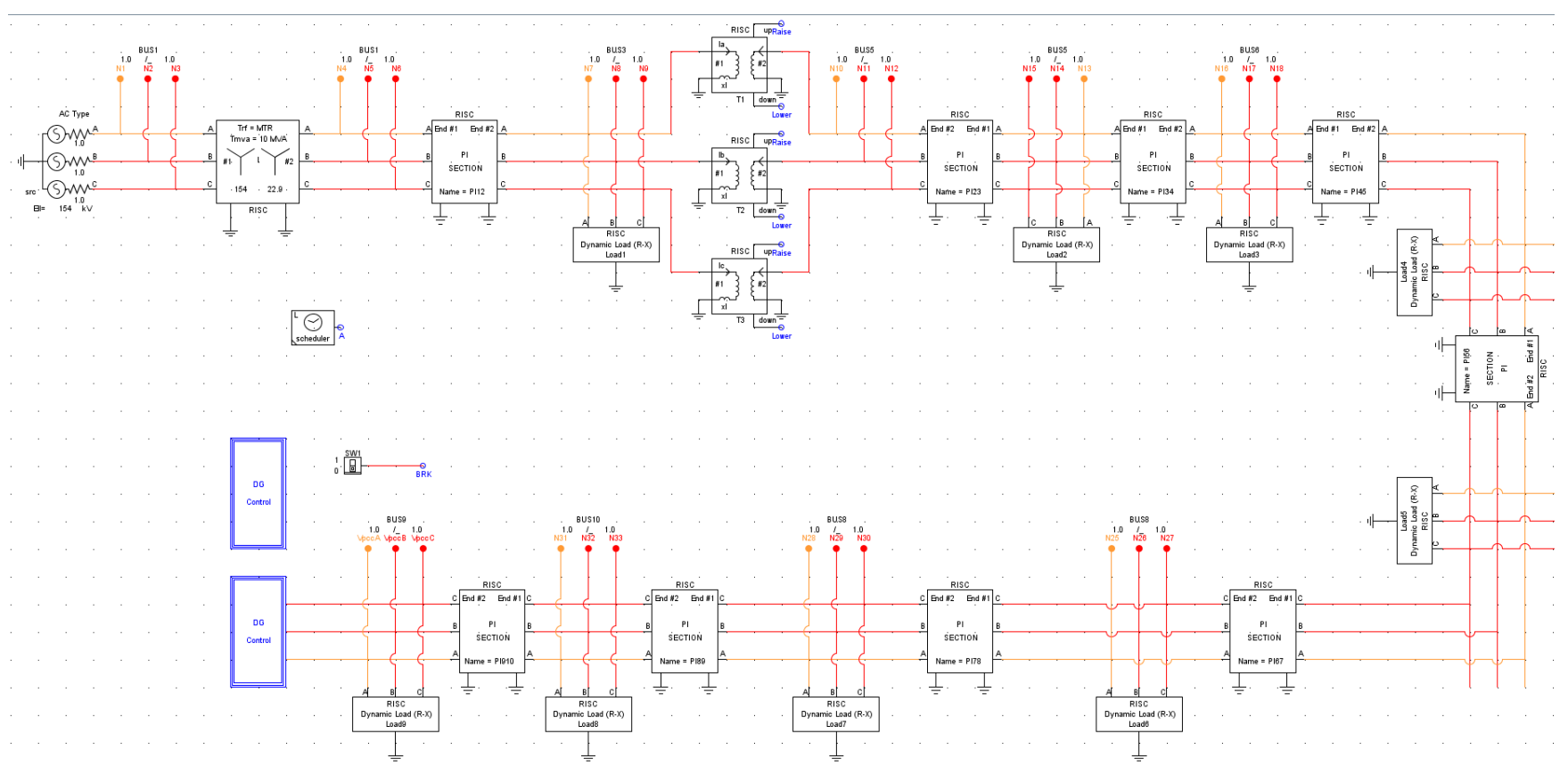

Fig. 8. The distribution system model in RTDS environment
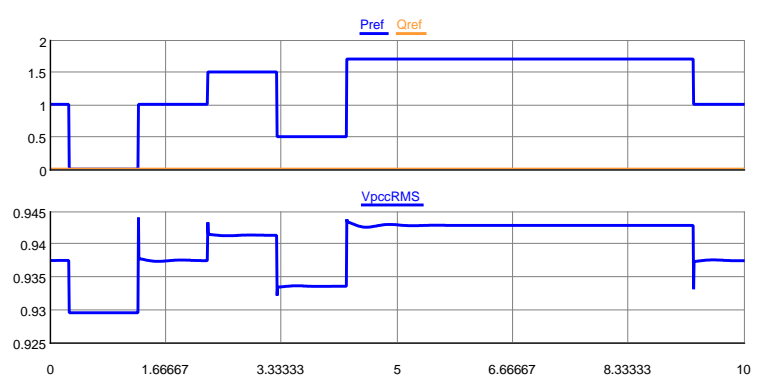

Fig. 9. The voltage change due to the change of active power.

From equation 6:

$$
\Delta V_{P C C} \approx 1 \times 0.0347 \times 18=0.6246 \%
$$

Comparing with result from Fig. 9, when $\mathrm{P}_{\mathrm{DG}}$ changes from 0 to $1 \mathrm{MW}$, the PCC voltage variation is $\Delta \mathrm{V}_{\mathrm{PCC}}=0.63 \%$. The error is just $0.0054 \%$.

Case 2: Change $Q_{D G}$ to keep $\Delta V_{P C C} \approx 0$.

From equation 5 , to keep $\Delta V_{P C C} \approx 0$, the reactive power of DG should be:

$$
\begin{aligned}
& \Delta V_{P C C} \approx P_{D G} R+Q_{D G} X=0 \\
& \rightarrow Q_{D G}=-\frac{R}{X} P_{D G}
\end{aligned}
$$
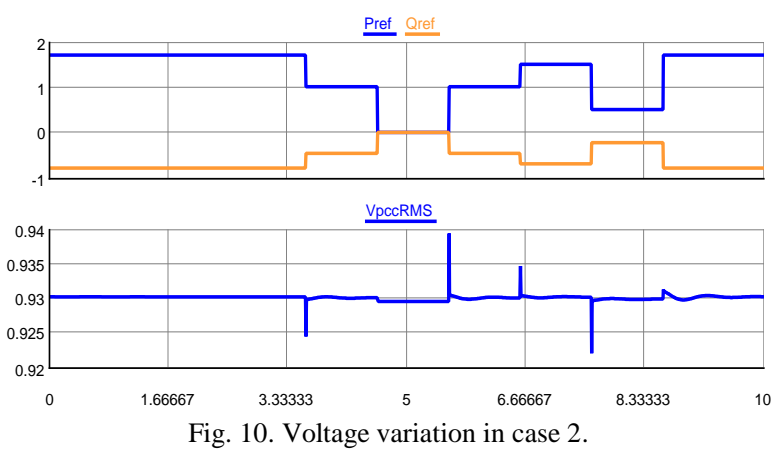

When PDG changes, QDG is calculated from equation (7) to keep the voltage variation at PCC nearly zero. The result of this case is shown in Fig. 10.
From the above results, it can be seen that the DG model can work well in case we want to change voltage at PCC follow reference value or keep voltage variation is constant. This simple DG model can be applied to many applications to simulate effect of DG in distribution system or even microgrid.

\section{B. Cooperative Control Scheme of BESS and DG}

The aim of this section is to develop a simple, aggregated model for testing the coordinated implementation of BESS and DG in distribution system. A. In this paper, with the purpose of testing the correctness of DG and BESS model, a simple cooperative control scheme is used to control power flows at the PCC.

Active power at the PCC is calculated from equation (8) as follows:

$$
P_{P C C}=P_{B E S S}+P_{D G}
$$

The objective is to keep the active power at the PCC the preplanned value when the output active power of DG changes by charging/discharging battery bank of BESS. From equation (8), with the desired value of the PCC active powerso called $\mathrm{P}_{\text {order }}$, the output active power of BESS can be obtained easily as follows:

$$
P_{B E S S}^{*}=P_{O R D E R}-P_{D G}
$$

The scheme is composed of two requirements. When the output of DG $P_{D G}$ is larger than $P_{\text {order }}$, BESS must be charged to absorb the surplus active power. In the contrary, BESS should be discharged to compensate the shortage. Therefore, by controlling the operating mode of BESS, the summation of the active power output $\left(P_{B E S S}+P_{D G}\right)$ always matches the requirement.

Fig. 11 shows the basic connection of BESS and DG at the PCC in RTDS environment.

For the simulation purposed in this paper, we are suggesting that BESS can satisfy some system requirements such as energy capacity, safety charge/discharge limits and so on. 


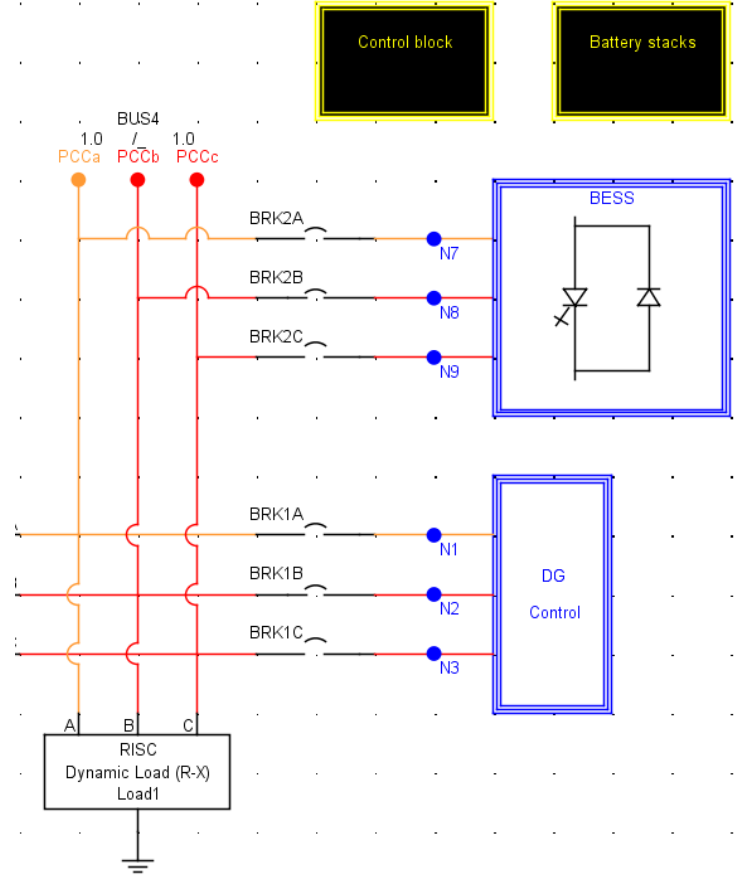

Fig. 11. BESS - DG cooperative control scheme at the PCC.

The reactive power control at the PCC can be determined in the same way with active power as required by the operators. However, this issue is not in the scope of this paper. In this paper the reactive power reference is simply set as zero.
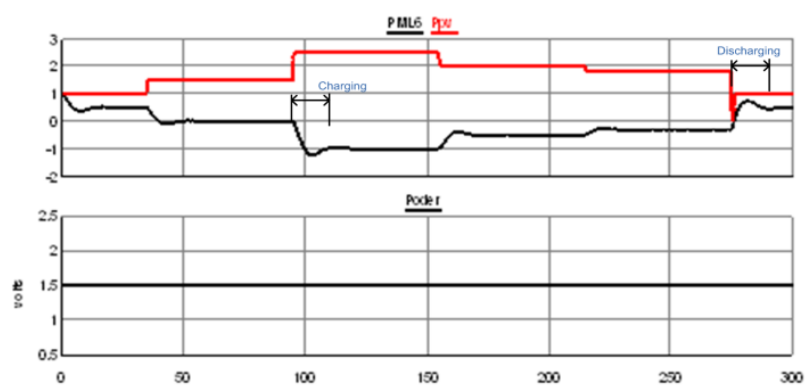

Fig. 12. Active power output control of BESS.

The result from Fig. 12 shows that, when the output of DG exceeds the desired value, the BESS is operated in charging mode to absorb the surplus amount, and vice versa. The example of charging/discharging period can be seen in Fig. 12 The minus '-' value means battery of BESS is in charging mode, and in the contrary, plus ' + ' value shows the discharging mode. The summation of output active power of BESS and DG at any time is always kept the constant.

\section{CONCLUSION}

This paper presents user defined models of DG and BESS by using mathematics components and Cbuilder software module in RTDS environment. Moreover, the control schemes of DGs and BESS to improve voltage and active power control performance were also proposed. The simulation results verified the implementation of each component and the developed UDM. It can be used to preliminary test the effect of DG and BESS controllers to voltage control problem in distribution systems before using actual devices. The proposed system and control scheme provides a solution to help achieve the effect of DG and BESS in distribution system.

\section{ACKNOWLEDGMENT}

This work was supported by Korea Institute of Energy Technology Evaluation \& Planning (KETEP) grant funded by Korea government Ministry of Trade, Industry \& Energy (No. 20123010020080) and the BK 21 PLUS project.

\section{REFERENCES}

[1] P. Piagi and R. H. Lasseter, "Microgrid: a conceptual solution," in Proc. Power Electronics Specialists Conf., Jun. 2004, vol. 6, pp. 4285-4290.

[2] L. A. Kojovic, "Coordination of distributed generation and step voltage regulator operations for improved distribution system voltage regulation," in Proc. Power eng. Soc. General Meeting, 2006.

[3] M. Kim, R. Hara, and H. Kita, "Design of the optimal ULTC parameters in distribution system with distributed generations," IEEE Trans. Power Syst., vol. 24, no. 1, pp. 297-305, 2009.

[4] S. White, "Active local distribution network management for embedded generation-GEN AVC," Department of Trade and Industrial, London, UK, 2005.

[5] F. A. Chacra, P. Bastard, G. Fleury, and R. Clavreul, "Impact of energy storage costs on economic performance in a distribution substation," IEEE Trans. Power Syst., vol. 20, no. 2, pp. 684-691, May 2005.

[6] T. T. Trung, Seon-Ju Ahn, and Joon-Ho Choi, "Real time simulation studies of distribution system with DGs", presented at Advance Power System Automation and Protection Conference, Jeju, Korea, 2013.

[7] H. Saadat, Power System Analysis, $2^{\text {nd }}$ ed., June 2002, pp. $19-23$.

[8] J. H. Jeon, J. Y. Kim, S. K. Kim, C. H. Cho et al., "Real time digital simulator based test system for microgrid management system," in Proc. Transmission \& Distribution Conference \& Exposition: Asia and Pacific, 2009, pp. 1-4.

[9] C. M. Shepherd, "Design of primary and secondary cells - part 2. an equation describing battery discharge," Journal of Electrochemical Society, vol. 112, pp. 657-664, July 1965.

[10] O. Tremblay and L. A. Dessaint, "Experimental validation of a battery dynamic model for EV applications," World Electric Vehicle Journal vol. 3, 2009.

[11] RTDS Technologies, Real Time Digital Simulation for the Power Industry - Manual Set.

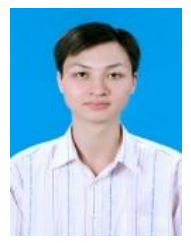

Tran Thai Trung is now a M.S student in Electrical Engineering Department, Chonnam National University, Gwangju, Korea. His research interests are distributed energy resources, distribution networks, signal processing and real-time simulation.

Seon-Ju Ahn received his B.S., M.S., and Ph.D. degrees in electrical engineering from Seoul National University, Seoul, Korea, in 2002, 2004, and 2009 , respectively. He was a postdoctoral researcher at Myongji University, Korea, and FREEDM System Center, NC State University, Raleigh, NC. Currently, He is an assistant professor at Chonnam National University, Gwangju, Korea. His research interests are power quality, distributed energy resources, microgrids, smart grids, and real-time simulation.

Joon-Ho Choi received the B.S., M.S. and Ph.D. degrees in electrical engineering from Soongsil University, Seoul, Korea in 1996, 1998 and 2002 , respectively. He was a BK21 post-doctoral fellow at Seoul National University. Currently, he is an associate professor at Chonnam National University, Gwang-ju, Korea. His interests include operation and integration strategies of distributed generation, distribution automation, and modeling and operation algorithms of the smart grid. 